\title{
Morphology of Optic Disc Through Heidelberg Retina Tomograph in Retinal Vein Occlusions Alone or in Combination with Primary Open Angle Glaucoma ${ }^{\S}$
}

\author{
Alessandro Guido Actis ${ }^{*}$, , Luca Belli $^{2}$, Laura Dall' orto ${ }^{1}$, Rachele Penna ${ }^{1}$, Beatrice Brogliatti ${ }^{1}$ and \\ Teresa Rolle ${ }^{1}$ \\ ${ }^{I}$ Department of Surgical Sciences, Eye Clinic and Doctoral School in Life and Health Sciences. University of Turin. \\ Italy \\ ${ }^{2}$ Department of Ophthalmology, Hospital of Ivrea, A.S.L. Torino 4, Italy
}

\begin{abstract}
Purpose: To evaluate the morphology of optic discs in eyes suffering from retinal vein occlusion (RVO) alone or in combination with primary open-angle glaucoma (POAG).

Methods: Prospective, observational study. 48 consecutive patients were enrolled, 30 with unilateral RVO diagnosis, 18 with unilateral retinal vein occlusion (RVO) associated with POAG. We divided RVOs on the basis of occlusion site: arterio-venous crossing (AV-RVO), optic cup (OC-RVO), optic nerve (ON-RVO) with head nerve swelling (ONHSRVO) or without it (NONHS-RVO). A control group of 25 patients who were sex and age matched was selected.

Results: Comparing the fellow eyes of the patients with RVO and control healthy eyes, no differences emerged in cup/disc ratio but they came out for the HRT values in Rim Area, cup shape measure and height variation contour $(\mathrm{p}<0.05)$.

The most frequent occlusion site was at the level of an arteriovenous crossing in patients not suffering from POAG $(36.7 \%)$ and at the level of the optic cup in patients with RVO and POAG (50\%). In the RVO group without POAG, the OC-RVO subgroup has shown an higher cup area $(0.366 \pm 0.094)$ and cup/disc area ratio $(0.184 \pm 0.063)$, a lower rim volume $(0.374 \pm 0.021)$ and a different cup shape measure $(-0.221 \pm 0.066)(\mathrm{p}<0.05)$ compared with the AV and NONHS sites. Compared with NONHS group differences emerged also for the fibres parameters and in the height variation contour $(0.346 \pm 0.081)$. Also in the RVO group with POAG significant differences $(\mathrm{p}<0.05)$ have been surveyed between OC-RVO and other occlusion sites in cup area $(0.119 \pm 0.029)$, cup/disc area ratio $(0.532 \pm 0.09)$, rim volume $(0.374 \pm 0.07)$, cup/shape measure $(-0.079 \pm 0.013)$.

Conclusions: Classification of the analyzed parameters on the basis of the occlusion site provides a basis for which clinical decisions and research on causal factors in future studies can be based on.
\end{abstract}

Keywords: Optic disc, retinal vein occlusion (RVO), glaucoma, HRT.

\section{INTRODUCTION}

Retinal vein occlusion (RVO), including both branch retinal vein occlusion (BRVO) and central retinal vein occlusion (CRVO), is a common sight-threatening retinal vascular disorder with significant morbidity [1]. Major causes of vision loss include macular edema and neovascularization with secondary vitreous hemorrhage and/or neovascular glaucoma [1].

Open-angle glaucoma is a progressive retinal ganglion cells degeneration characterized by a specific pattern of

*Address correspondence to this author at the c/o Ospedale Oftalmico di Torino, Via Juvarra 19, 10121, Torino, Italy; Tel: +39 392 2259680; Fax: +39 (0)11 539024; E-mail: alessandro.actis@gmail.com

${ }^{\S}$ The study has been conducted in the Department of Surgical Sciences Eye Clinic - University of Turin, Italy.

This manuscript has been conducted with ethical standards, it follows all principles approved in the Declaration of Helsinki and its later amendments, it has been conducted with informed consent of all the subjects. visual field and optic nerve head damage [2,3]. Clinical trials confirmed the importance of intraocular pressure (IOP) in the development and progression of open-angle glaucoma, even if evidence suggests the existence of ocular and systemic factors, in addition to IOP, that can be responsible of this development and progression [4].

An association between primary open angle glaucoma (POAG) or ocular hypertension (OHT) and RVO has been found in many studies [5]. Many authors have underlined this association on the basis of different etiopathogenetic theories. In their review, Sonnsjio and Krakau [6] observed an high incidence of RVOs, both central and branch, in a wider group of patients suffering from POAG. According to these authors, the little flame shaped peripapillary hemorrhages, usually seen in patients suffering from glaucoma, correspond to little vein occlusions. The coexistence of these signs suggests a common aetiology, a hypothesis that has always gained great popularity supported by the vascular theory at the basis of the glaucoma damage [7]. Beside the vascular component, many studies have 
suggested that alterations of the normal anatomy of the optic nerve head could cause a site particularly susceptible to the vein occlusion.

So it can be useful to analyze the optic disc morphologic features in subjects suffering from a retinal vein occlusion, also combined with the diagnosis of primary open-angle glaucoma, with the aim to understand if some anatomic features are associated or not to a determinate occlusion site.

Purposes of our study are:

- $\quad$ to evaluate morphology of the optic disc in eyes suffering from RVO also combined with the diagnosis of POAG;

- to search possible differences in the optic disc parameters studied through the imaging supplied by the HRT II (Heidelberg Retina Tomograph Heidelberg Engineering - Germany) between the fellow eyes suffering from RVO (not suffering from glaucoma) and a control group of healthy eyes; between the different occlusion sites and finally to study optic disc morphology in the group of patients with papillary edema;

- $\quad$ to define if some morphologic features of the optic disc can or not suggest an etiopathogenetic relation with the retinal vein occlusions considering both the occlusion site and the POAG presence.

\section{MATERIALS AND METHODOLOGY}

The study has been carried out on a group of patients with retinal vein occlusion diagnosis sent to our Ophthalmologic Clinic in the last 5 years.

The study has been conducted with ethical standards, it followed all principles approved in the Declaration of Helsinki and its later amendments, it has been conducted with written informed consent of all the subjects.

48 consecutive patients with diagnosis of unilateral RVO have been included. 18 among them presented a positive clinical history for POAG.

Patients were classified as having POAG when they had a typical glaucomatous visual field, possibility of typical abnormal optic nerve head (before RVO diagnosis), open angle at gonioscopy, IOP $>21 \mathrm{mmHg}$ with no treatment and no clinically apparent secondary cause for their glaucoma [2,3]. Visual fields have been assessed by an Humphrey Field Analyzer 750 (HFA, Humphrey, Inc, San Leandro, CA, USA), 24-2 SITA (Swedish Interactive Threshold Algorithm) standard. A glaucomatous visual field defect was defined as:

- $\quad$ three adjacent points depressed by $5 \mathrm{~dB}$, with one of the points depressed by at least $10 \mathrm{~dB}$ or

- $\quad$ two adjacent points depressed by $10 \mathrm{~dB}$ or

- $\quad 10 \mathrm{~dB}$ difference across the nasal horizontal meridian in two adjacent points.

None of the points could be edge points unless immediately above or below the nasal horizontal meridian. In addition, visual field testing was considered reliable only when false-negative and false-positive responses were less than $30 \%$ and fixation losses were less than $20 \%$ on HFA.

Of the 48 patients included in the study, in 30 patients we diagnosed unilateral RVO and in 18 patients we diagnosed unilateral RVO and positive clinical history of POAG. We selected a control group of 25 healthy subjects (analysis of 25 eyes), age and sex matched to the group of patients with unilateral retinal vein thrombosis without POAG.

Occlusions of the venous drainage of the retina classically have been divided into CRVO and BRVO. More recently RVOs that involve approximately half of the retina have been recognised as a distinct type of RVOs, but there is still confusion as to wheter this group are variants of BRVO or CRVO. We decided, according to Beaumont [8], to define three anatomic occlusion sites on the basis of the fluorescein angiography aspect: arteriovenous crossing (AV-RVO), optic cup (OC-RVO) and optic nerve (ON-RVO). In case of occlusion at the level of an arteriovenous crossing or of the optic cup, the point where the vein calibre changes, is well visible, unless it is obscured by hemorrhages, exudates or retinal edema. The occlusions localized at the optic nerve level have been identified by the fact that the vein enters in a dilated form into the nervous fibers. They have been subdivided in two categories, on the basis of the presence (Optic Nerve Head Swelling; ONHS-RVO) or not (Non Optic Nerve Swelling; NONHS-RVO) of optic nerve head $(\mathrm{ONH})$ edema. The ONH edema is defined as a swelling of the optic nerve compared to the surrounding retina, associated to edema, exudates and hemorrhages. The simple presence of blurred margins without disc swelling was not considered a sufficient sign to include the case in the group with $\mathrm{ONH}$ edema.

We excluded from our study those uncommon retinal occlusions without a precise anatomic reference point, defined "no site" RVO (7 patients). We excluded also: patients that had ocular surgery or trauma in the previous year before the study; patients that had ocular infections within 3 months before the study; pregnant or nursing women; inability to understand and/or complete the informed consent and the study; patients less than 18 years old.

All patients have been subjected to:

- complete eyes examination with measure of the intraocular pressure with Goldmann Applanation Tonometer (GAT). The IOP measure was carried out between 8:00 a.m. and 10:00 a.m.;

- fluorescein angiography test through H.R.A. (Heidelberg Retina Angiograph);

- morphologic test of the optic nerve head through HRT II (Heidelberg Retina Tomograph II).

The HRT II is a confocal laser scanning microscope which allows the acquisition and the analysis of threedimensional images of the retinal surface at multiple focal planes. The laser source used for the scanning is a diodeslaser with wave length of $670 \mathrm{~nm}$. Using confocal scanning principles, a 3-dimensional topographic image is constructed from a series of optical image sections at consecutive focal planes. The stereometric analysis of the images gives a set of parameters which describe the optic nerve head. Iester et al. 
[9] noted a statistically significant correlation (Pearson's r, p $<0.001$ ) between cup area, cup/disc area ratio, rim area, rim volume, cup shape measure, and retinal nerve fibre layer cross-section area with Humphrey's visual field (Carl Zeiss Meditec, Dublin, California) mean deviation (MD) and corrected pattern standard deviation (CPSD). Multiple linear regression analysis demonstrated that rim area was the most important predictor of mean deviation and pattern standard deviation (PSD).

For the group of patients suffering from optic nerve vein occlusion with papilla edema (ONHS-CRVO) we have used the prominence analysis protocol of the HRT software because the papilla swelling on the retinal plane doesn't allow the use of the standard processing.

A paired $t$ test between RVO and RVO associated with POAG analyzed differences in the occlusion site frequency. The comparison among the HRT parameters has been obtained with Student's $t$ test and repeated-measures of ANOVA. The value of $p<0.05$ was considered statistically significant.

\section{RESULTS}

Of the 48 patients included in the study;

- $\quad$ in 30 patients we diagnosed unilateral RVO (30 eyes), 17 males $(56,6 \%)$ and 13 females $(43,3 \%)$ with mean age of $62,3 \pm 10,9$ years. The average IOP of this group was equal to $15,5 \pm 1,8 \mathrm{mmHg}$ (GAT).

- $\quad$ in 18 patients we diagnosed unilateral RVO and positive clinical history for POAG, 10 males $(55,5 \%)$ and 8 females $(44,4 \%)$, mean age of $68,5 \pm 7,4$ years. The average IOP of this group was $18,8 \pm 2,0 \mathrm{mmHg}$ (GAT).

We selected a control group of 25 healthy subjects (analysis of 25 eyes), 15 males $(60 \%)$ and 10 females (10\%) with average age $62,9 \pm 4,7$ years, age and sex matched to the group of patients with unilateral retinal vein thrombosis without POAG. The average IOP of this control group was $15,1 \pm 1,7 \mathrm{mmHg}$.

The features of the patients included in the study have been summed up in Tables $\mathbf{1}$ and $\mathbf{2}$.

\section{Comparison Between RVO-Group and Healthy Subjects}

We compared the 30 contralateral eyes (fellow eye) of the patients suffering from RVO without association of POAG with the 25 eyes of healthy subjects, homogeneous for sex and age.

In Table 3 the results of the HRT parameters comparison are shown by means of the Student's test and repeated measures of ANOVA.

\section{Comparison of HRT Parameters in Relation to Occlusion Sites}

We studied the correlation between the HRT parameters in relation to the occlusion site (Tables $\mathbf{4}$ and $\mathbf{5}$ ).

In Table $\mathbf{4}$ we note a statistically significant difference $(\mathrm{p}<0.05)$ in four parameters in the comparison between the

Table 1. Features of the Patients Included in the Study

\begin{tabular}{|c|c|c|c|}
\hline & RVO & RVO Associated with POAG & Healthy Controls \\
\hline Eyes & 30 & 18 & 25 \\
\hline Age (years \pm SD) & $62.3 \pm 10.9$ & $68.5 \pm 7.4$ & $62.9 \pm 4.7$ \\
\hline Gender & $\begin{array}{l}17 \text { males }(56,6 \%) \text {, } \\
13 \text { females }(43,3 \%)\end{array}$ & $\begin{array}{l}10 \text { males }(55,5 \%) \text {, } \\
8 \text { females }(44,4 \%)\end{array}$ & $\begin{array}{l}15 \text { males }(60 \%) \text {, } \\
10 \text { females }(40 \%)\end{array}$ \\
\hline Mean IOP $(\mathrm{mmHg} \pm \mathrm{SD})$ & $15.5 \pm 1.82$ & $18.8 \pm 2.0$ & $15.1 \pm 1.7$ \\
\hline OC-RVO (occlusion at the optic disc level) & $6(20 \%)$ & $9(50 \%)$ & --- \\
\hline AV-RVO (occlusion at the level of the arterovenous crossing ) & $11(36.7 \%)$ & $2(11.1 \%)$ & --- \\
\hline ONHS-RVO (occlusion at the level of the optic nerve with disc edema) & $8(26.7 \%)$ & $4(22.2 \%)$ & --- \\
\hline NONHS -RVO (occlusion at the level of the optic nerve without disc edema) & $5(16.6 \%)$ & $3(16.7 \%)$ & --- \\
\hline
\end{tabular}

RVO: Retinal Vein Occlusion; POAG: Primary Open Angle Glaucoma; AV-RVO: Artero-Venous crossing Retinal Vein Occlusion; OC-RVO: Optic Cup Retinal Vein Occlusion; ON-RVO: Optic Nerve Retinal Vein Occlusion with Head Nerve Swelling (ONHS-RVO) or without it (NONHS-RVO).

Table 2. Mean IOP and Age Per Occlusion Site

\begin{tabular}{|c|c|c|c|c|}
\hline & RVO $(\mathbf{n = 3 0})$ & RVO and POAG (n=18) & Mean IOP (mmHg \pm SD) & Mean Age (Years \pm SD) \\
\hline \hline OC-RVO & 6 & 9 & $18.9 \pm 5.2$ \\
\hline AV-RVO & 11 & 2 & $15.5 \pm 2.2$ & $16.2 \pm 12.4$ \\
\hline ONHS-RVO & 8 & 4 & $3.2 \pm 10.5$ & $5.4 \pm 7.9$ \\
\hline NONHS-RVO & 5 & 3 & $16.7 \pm 2.8$ \\
\hline
\end{tabular}

RVO: Retinal Vein Occlusion; POAG: Primary Open Angle Glaucoma; AV-RVO: Artero-Venous crossing Retinal Vein Occlusion; OC-RVO: Optic Cup Retinal Vein Occlusion; ON-RVO: Optic Nerve Retinal Vein Occlusion with Head Nerve Swelling (ONHS-RVO) or without it (NONHS-RVO). 
Table 3. HRT II Parameters (Mean Values \pm SD): Comparison Between Fellow Eye RVO and Healthy Control Group

\begin{tabular}{|l|c|c|c|}
\hline & Fellow Eye RVO & Controls \\
\hline \hline Disk area $\left(\mathrm{mm}^{2} \pm \mathrm{sd}\right)$ & $1.997 \pm 0.865$ & $2.121 \pm 0.973$ & 0.112 \\
\hline Cup area $\left(\mathrm{mm}^{2} \pm \mathrm{sd}\right)$ & $0.481 \pm 0.194$ & $0.411 \pm 0.183$ & 0.08 \\
\hline Cup disk area ratio & $0.216 \pm 0.102$ & $0.188 \pm 0.091$ & 0.291 \\
\hline Rim area $\left(\mathrm{mm}^{2} \pm \mathrm{sd}\right)$ & $1.517 \pm 0.342$ & $0.252 \pm 0.065$ & 0.003 \\
\hline Height variation contour $(\mathrm{mm} \pm \mathrm{sd})$ & $0.351 \pm 0.081$ & $0.056 \pm 0.012$ & 0.001 \\
\hline Cup volume $\left(\mathrm{mm}^{3} \pm \mathrm{sd}\right)$ & $0.109 \pm 0.044$ & $0.458 \pm 0.113$ \\
\hline Rim volume $\left(\mathrm{mm}^{3} \pm \mathrm{sd}\right)$ & $0.375 \pm 0.095$ & $0.112 \pm 0.025$ \\
\hline Mean cup depth $(\mathrm{mm} \pm \mathrm{sd})$ & $0.175 \pm 0.063$ & $0.479 \pm 0.132$ \\
\hline Maximum cup depth $(\mathrm{mm} \pm \mathrm{sd})$ & $0.502 \pm 0.142$ & $-0.329 \pm 0.051$ & 0.171 \\
\hline Cup shape measure & $-0.226 \pm 0.043$ & $0.171 \pm 0.114$ & 0.08 \\
\hline Mean RNFL thickness $(\mathrm{mm} \pm \mathrm{sd})$ & $0.215 \pm 0.141$ & $0.882 \pm 0.253$ \\
\hline RNFL cross sectional area $\left(\mathrm{mm}{ }^{2} \pm \mathrm{sd}\right)$ & $1.073 \pm 0.965$ & 0.0025 \\
\hline
\end{tabular}

RVO: Retinal Vein Occlusion; POAG: Primary Open Angle Glaucoma; RNFL: Retinal Nerve Fiber Layer.

Three parameters (Rim Area, Height Variation Contour and Cup Shape Measure) reached a statistically significant difference (p<0.05).

OC group and the AV group: Cup Area, Cup/Disc Ratio, Rim Volume and Cup Shape Measure. We note a statistically significant difference in seven parameters comparing the $\mathrm{OC}$ group and the NONHS group: Cup Area, Cup/Disc ratio, Height Variation Contour, Rim Volume, Cup Shape Measure, Mean RNLF thickness and RNFL Cross Sectional Area (CSA).

In Table $\mathbf{5}$ we note a statistically significant difference similar to the previous one concerning the comparison between the OC group and the AV group. We note a significant difference in five parameters in the comparison between the OC group and the NONHS group: Cup Area, Cup/Disc ratio, Rim Volume, mean Cup Depth and Cup Shape Measure.
In Figs. $(\mathbf{1}, \mathbf{2})$ the frequency of the occlusion site is depicted in the groups without or associated with POAG, respectively.

\section{Analysis of ONHS-CRVO Group}

In the group of patients suffering from vein occlusion at the optic nerve level with $\mathrm{ONH}$ edema, we have used the prominence analysis protocol of the HRT software (Table 6).

Data of this protocol supply a good description of the typical ONH edema that, among other things, can not be studied in their initial features because deeply altered from the anatomic point of view.

This typical ONH swelling (edema) is the result of the axonal transport blockage at the level of the cribrous lamina

Table 4. HRT II Parameters (Mean Values \pm SD) Per Occlusion Site in the RVO Group

\begin{tabular}{|c|c|c|c|c|c|}
\hline & OC - RVO & AV-RVO & $\begin{array}{c}P \\
O C v s A V\end{array}$ & NONHS-RVO & $\begin{array}{c}P \\
\text { OC vs NONHS }\end{array}$ \\
\hline Disk area $\left(\mathrm{mm}^{2} \pm \mathrm{sd}\right)$ & $2.029 \pm 0.914$ & $1.937 \pm 0.865$ & 0.15 & $1.886 \pm 0.943$ & 0.17 \\
\hline Cup area $\left(\mathrm{mm}^{2} \pm \mathrm{sd}\right)$ & $0.366 \pm 0.094$ & $0.228 \pm 0.083$ & 0.01 & $0.209 \pm 0.077$ & 0.016 \\
\hline Cup disk area ratio & $0.184 \pm 0.063$ & $0.117 \pm 0.042$ & 0.005 & $0.111 \pm 0.012$ & 0.007 \\
\hline Rim area $\left(\mathrm{mm}^{2} \pm \mathrm{sd}\right)$ & $1.664 \pm 0.94$ & $1.709 \pm 0.651$ & 0.12 & $1.667 \pm 0.594$ & 0.15 \\
\hline Height variation contour $(\mathrm{mm} \pm \mathrm{sd})$ & $0.346 \pm 0.081$ & $0.443 \pm 0.072$ & 0.07 & $0.502 \pm 0.045$ & 0.01 \\
\hline Cup volume $\left(\mathrm{mm}^{3} \pm \mathrm{sd}\right)$ & $0.055 \pm 0.016$ & $0.043 \pm 0.018$ & 0.29 & $0.033 \pm 0.012$ & 0.45 \\
\hline Rim volume $\left(\mathrm{mm}^{3} \pm \mathrm{sd}\right)$ & $0.374 \pm 0.021$ & $0.503 \pm 0.043$ & 0.005 & $0.547 \pm 0.052$ & 0.003 \\
\hline Mean cup depth (mm \pm sd) & $0.144 \pm 0.012$ & $0.136 \pm 0.017$ & 0.752 & $0.132 \pm 0.018$ & 0.565 \\
\hline Maximum cup depth (mm \pm sd) & $0.456 \pm 0.103$ & $0.521 \pm 0.145$ & 0.07 & $0.517 \pm 0.136$ & 0.09 \\
\hline Cup shape measure & $-0.221 \pm 0.066$ & $-0.302 \pm 0.08$ & 0.006 & $-0.361 \pm 0.091$ & 0.004 \\
\hline Mean RNFL thickness (mm $\pm s d)$ & $0.207 \pm 0.069$ & $0.245 \pm 0.076$ & 0.065 & $0.291 \pm 0.075$ & 0.01 \\
\hline RNFL cross sectional area $\left(\mathrm{mm}^{2} \pm \mathrm{sd}\right)$ & $1.043 \pm 0.091$ & $1.256 \pm 0.188$ & 0.11 & $1.419 \pm 0.245$ & 0.02 \\
\hline
\end{tabular}

RVO: Retinal Vein Occlusion; POAG: Primary Open Angle Glaucoma; RNFL: Retinal Nerve Fiber Layer; AV-RVO: Artero-Venous crossing Retinal Vein Occlusion; OC-RVO: Optic Cup Retinal Vein Occlusion; ON-RVO: Optic Nerve Retinal Vein Occlusion without Head Nerve Swelling (NONHS-RVO). 
Table 5. HRT II Parameters (Mean Values \pm SD) Per Occlusion Site in the RVO and POAG Group

\begin{tabular}{|c|c|c|c|c|c|}
\hline & OC - RVO & AV-RVO & $\begin{array}{c}P \\
O C v s A V\end{array}$ & NONHS-RVO & $\begin{array}{c}P \\
\text { OC vs NONHS }\end{array}$ \\
\hline Disk area $\left(\mathrm{mm}^{2} \pm \mathrm{sd}\right)$ & $2.102 \pm 0.876$ & $1.987 \pm 0.912$ & 0.18 & $1.956 \pm 0.763$ & 0.157 \\
\hline Cup area $\left(\mathrm{mm}^{2} \pm \mathrm{sd}\right)$ & $0.119 \pm 0.029$ & $0.787 \pm 0.063$ & 0.004 & $0,765 \pm 0.061$ & 0.003 \\
\hline Cup disk area ratio & $0.532 \pm 0.09$ & $0.396 \pm 0.077$ & 0.02 & $0.391 \pm 0.084$ & 0.035 \\
\hline Rim area $\left(\mathrm{mm}^{2} \pm \mathrm{sd}\right)$ & $0.983 \pm 0.104$ & $1.201 \pm 0.187$ & 0.13 & $1.191 \pm 0.163$ & 0.27 \\
\hline Height variation contour $(\mathrm{mm} \pm \mathrm{sd})$ & $0.473 \pm 0.081$ & $0.534 \pm 0.097$ & 0.25 & $0.513 \pm 0.094$ & 0.36 \\
\hline Cup volume $\left(\mathrm{mm}^{3} \pm \mathrm{sd}\right)$ & $0,498 \pm 0.094$ & $0.436 \pm 0.087$ & 0.59 & $0.467 \pm 0.079$ & 0.473 \\
\hline Rim volume $\left(\mathrm{mm}^{3} \pm \mathrm{sd}\right)$ & $0.374 \pm 0.07$ & $0.554 \pm 0.086$ & 0.005 & $0.523 \pm 0.091$ & 0.0045 \\
\hline Mean cup depth (mm \pm sd) & $0.513 \pm 0.101$ & $0.493 \pm 0.098$ & 0.004 & $0.378 \pm 0.103$ & 0.006 \\
\hline Maximum cup depth $(\mathrm{mm} \pm \mathrm{sd})$ & $0.922 \pm 0.143$ & $0.876 \pm 0.107$ & 0.08 & $0.798 \pm 0.123$ & 0.13 \\
\hline Cup shape measure & $-0.079 \pm 0.013$ & $-0.167 \pm 0.092$ & 0.013 & $-0.154 \pm 0,084$ & 0.016 \\
\hline Mean RNFL thickness (mm \pm sd) & $0.187 \pm 0.096$ & $0.199 \pm 0.056$ & 0.75 & 0.178 & 0.432 \\
\hline RNFL cross sectional area $\left(\mathrm{mm}^{2} \pm \mathrm{sd}\right)$ & $1.043 \pm 0.141$ & $1.167 \pm 0.164$ & 0.69 & $1.123 \pm 0.094$ & 0.475 \\
\hline
\end{tabular}

RVO: Retinal Vein Occlusion; POAG: Primary Open Angle Glaucoma; RNFL: Retinal Nerve Fiber Layer; AV-RVO: Artero-Venous crossing Retinal Vein Occlusion; OC-RVO Optic Cup Retinal Vein Occlusion; ON-RVO: Optic Nerve Retinal Vein Occlusion without Head Nerve Swelling (NONHS-RVO).

or immediately behind. In the CRVOs it indicates that the vein occlusion has caused a serious ischemia in the retrolaminar region with complete blockage of the axoplasmic flow.

\section{DISCUSSION}

\section{Comparison Between RVO-Group and Healthy Subjects}

Several studies in literature tried to study a possible relationship between disc morphology and RVO [9-12].

At the basis of these studies there was the idea to compare the fellow eye of subjects suffering from vein occlusions with a control group of healthy eyes. These studies concluded that there was no statistically significant difference between the two groups and, for this reason, that the cup/disc ratio can't be predictive of a RVO development.

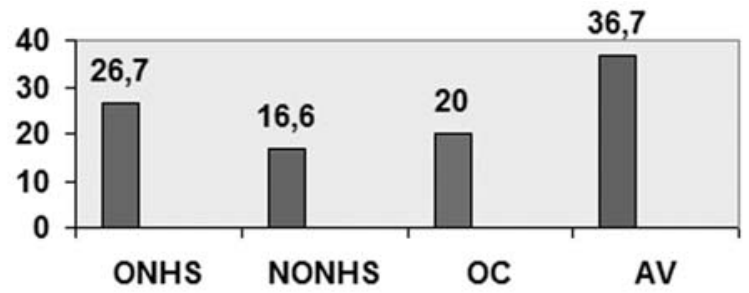

Fig. (1). Frequency of the occlusion sites of RVO group without POAG.

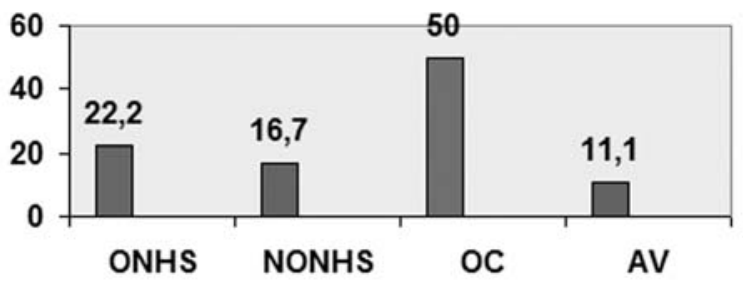

Fig. (2). Frequency of the occlusion sites of RVO group with POAG.
Table 6. HRT II Parameters (Mean Values \pm SD) of the Prominence Analysis in the ONHS-RVO Group

\begin{tabular}{|l|c|}
\hline \multicolumn{1}{|c|}{ Area $\left(\mathrm{mm}^{2}\right)$} & $\mathbf{1 . 8 7 5}$ \\
\hline \hline Area aboved curved surface $\left(\mathrm{mm}^{2}\right)$ & 0.776 \\
\hline Area above reference $\left(\mathrm{mm}^{2}\right)$ & 1.626 \\
\hline Mean radius $(\mathrm{mm})$ & 0.773 \\
\hline Mean height of contour $(\mathrm{mm})$ & -0.131 \\
\hline Height variation contour $(\mathrm{mm})$ & 0.567 \\
\hline Volume below reference $\left(\mathrm{mm}^{3}\right)$ & 0.036 \\
\hline Volume above reference $\left(\mathrm{mm}^{3}\right)$ & 0.601 \\
\hline Volume below surface $\left(\mathrm{mm}^{3}\right)$ & 0.192 \\
\hline Volume above surface $\left(\mathrm{mm}^{3}\right)$ & 0.044 \\
\hline Mean height in contour $\left(\mathrm{mm}^{3}\right)$ & 0.023 \\
\hline Effective mean height $\left(\mathrm{mm}^{2}\right)$ & 0.053 \\
\hline Maximun height in contour $(\mathrm{mm})$ & 0.139 \\
\hline Third moment in contour & -0.109 \\
\hline Mean variability & 0.033 \\
\hline
\end{tabular}

A recent study [13] has compared the diameters and disc area of RVO fellow eyes with optical coherence tomography (OCT), concluding that optic disc parameters in RVO fellow eyes were not different from normal subjects.

Recently data of the Ocular Hypertension Treatment Study (OHTS) [14] have been analysed. Twenty-six RVOs 5 branch, 14 central, and 7 hemicentral RVOs - were confirmed in 23 participants $(15$ observation and 8 medication). The 10-year cumulative incidence of RVO was $2.1 \%$ in the observation group and $1.4 \%$ in the medication group. At baseline, participants who later developed a RVO 
were significantly older and had greater vertical cup-to-disc ratios.

Furthermore in a recent paper Citirik et al. [15] investigated optic disk topography parameters of patients with BRVO using HRT. A relationship between BRVO and various optic disk parameters was found. They found optic disc parameters to differ significantly between affected and unaffected fellow eyes or control eyes but not between unaffected fellow eyes and control eyes.

In our study, comparing fellow eyes of patients with RVO with control healthy eyes, no statistically significant differences emerged concerning the cup/disc ratio. Three HRT parameters showed a statically significant difference.

The fellow eyes of the patients suffering from RVO presented a smaller rim area in comparison with the healthy ones, a wider variation range of the contour line profile height and a significant difference of the cup shape measure, parameter which expresses the overall form of the excavation.

The great difference in the cup shape measure indicates that these discs present a different spatial configuration when compared to normal discs and this is surely stimulating for further studies.

\section{Comparison of HRT Parameters in Relation to Occlusion Sites}

The importance to subdivide vein occlusions on the basis of the occlusion site and to study the features of the $\mathrm{ONH}$ with regard to this aspect is due to Beaumont $[8,16]$.

He concluded that optic cup and optic nerve-sited RVO without ONHS are associated with raised IOP. In contrast, glaucomatous optic disc cupping seems to be important in the OC-RVO group only. IOP, optic disc cupping and POAG diagnosis do not seem significantly linked to the development of $\mathrm{AV}-\mathrm{RVO}$ or when the occlusion occurs within the optic nevre in association with ONHS [8].

The classification of the RVOs on the basis of the occlusion site separates some groups which are different in a significant way for some morphologic features and for ocular variables.

As deducible from Table 1, the most frequent occlusion site in the not glaucomatous patients group is at the level of an arteriovenous crossing $(\mathrm{p}<0.05)$ while this site is the less frequent in the group of patients suffering from glaucoma.

The diagnosis of occlusion at the level of this site is very easy with fluorescein angiography. A thickened arteriolar wall can compress and narrow the vein lumen at the level of a crossing, predisposing to a RVO. Hence, the factors which cause pathology of the arteriolar wall in a selective way should be those closely associated to the occlusion in this site. Already Beaumont has established a relationship between the arterial hypertension and the cigarette smoke with RVO [8]. In general the risk factors for RVO are similar to those for cardiovascular pathologies [17, 18].

The mean IOP in the group of patients suffering from AV-RVO was $15,5 \pm 2,2 \mathrm{mmHg}$ which was not statistically significant when compared to the control group of healthy eyes (mean IOP $15,1 \pm 1,7 \mathrm{mmHg}$ ).
In the group of patients suffering from POAG the most frequent occlusion site is at the level of the optic cup (OCRVO) ( $\mathrm{p}<0.05)$ (Table 1). It could sometimes be difficult to make a differential diagnosis with the optic nerve occlusions without papilla edema since the hemorrhages can obscure the exact occlusion site. In these cases the aspect of the papillary vein, where it crosses the optic disc floor, is important to carry out a correct diagnosis. A papillary vein narrowed in this site represents a OC-RVO while a dilated vein suggests an occurred occlusion inside the nerve itself. The mean IOP level in the group OC-RVO was $18,9 \pm 5,2 \mathrm{mmHg}$, higher $(p<0.05)$ in comparison with the healthy controls and with all other subgroups of occlusion.

The close association between POAG, IOP increase and OC-RVO is quite predictable.

The OC-RVO group is that with the higher mean age (71,2 years followed by the group AV RVO with 68,2 years). The age increase is associated also to the higher IOP values and to the POAG incidence increase as deducible from literature $[2,3]$.

In the RVO group without POAG, the subgroup OCRVO has shown several HRT parameters altered in a significant way in comparison with the other subgroups: a higher cup area and cup/disc area ratio, a lower rim volume and a different cup shape measure in comparison with the AV and NONHS sites. In particular, in comparison with the NONHS group, an important difference occurred also for the fibres parameters and the height variation contour.

Also in the RVO group with POAG significant differences have been surveyed in the HRT parameters between the OC-RVOs and the other occlusion sites.

Hayreh [19] didn't recognize that a vein occlusion at the disc level can represent a different entity from other occlusions. Many years later Beaumont [8] proved that a diagnosis of optic disc occlusion can be made and has a great clinical utility. Lindblom [20] studied the incidence of vein occlusions in patients with POAG diagnosis remarking that the occlusion localizes, almost without exceptions, at the optic disc level. In a study with more than 10 years of follow up Lindbolm himself excluded an association between AVRVO and POAG.

In 1957 Dobree [21] was the first to describe the occlusion mechanism of a vein at the optic disc level in case of glaucoma. A wide pot-excavation implies a stretching and a weakening of the vein wall which loses the support and the protection of the optic nerve tissue becoming more susceptible to the mechanical stroke of a high IOP.

For this reason, we believe that the OC-RVO occurrence requires a very good tonometric compensation and imposes a further reduction of the target IOP previously estimated for that patient. This protects the patient also from a contralateral occlusion.

In the NONHS-CRVO group the main features are the incoming dilated vein in the nervous tissue, the absence of edema and of papilla swelling on the retinal plane. The average IOP level in this group didn't show differences with the control group $(p<0.05)$. The HRT parameters were not significantly different compared to the AV group, while they showed a lot of differences with the OC group. 
Beaumont [8] found a weak association between this form and POAG, supposing that in this disease a backwards displacement of the lamina cribrosa occurs. The lamina cribrosa should be the site where the vein occludes to determine the clinical manifestation. The misalignment of the lamina holes would narrow and angle the vessels through the lamina itself. Our survey included a limited number of patients in whom these associations are not evident. Nevertheless, the optic disc diameter was smaller in comparison with all the subgroups, even not reaching statistical significance in all parameters (Tables $\mathbf{4}$ and $\mathbf{5}$ ). A narrowing of the scleral channel could have a role with the occlusion at the lamina cribrosa level. Also in this group we believe that a frequent measurement of the IOP is important, reducing further the estimated target IOP.

\section{Analysis of ONHS-CRVO Group}

At the end of the discussion it worth to mention the cases of optic nerve head swelling (ONHS) in which we used the HRT prominence analysis protocol. In this group mean age was inferior in comparison with the other groups.

Many authors wrote different definitions and terms for these forms [22], better known as "papillophlebitis". The terminology suggests a phlogosis of the optic nerve or of the vein in the nerve context, even if significant evidences supporting this hypothesis have not been found yet.

\section{CONCLUSIONS}

Our study has got several limitations, particularly a small group of patients. This study will be widened in the involved patients number, using also other methods to evaluate disc morphology.

Our study highlighted how fundamental is to subdivide and classify the RVOs on the basis of the occlusion site. The particular anatomic site of the occlusion determines which factors, like a pathology of the vascular wall and the IOP, have a significant effect on the wall of the vein in that point. While the more frequent occlusion site is the arteriovenous crossing in the non glaucomatous eyes suffering from RVO, the occlusion is localized at the level of the optic disc excavation in half of the glaucomatous eyes of this study. In these occlusions we have observed the presence of several altered HRT morphologic parameters beside a higher IOP level. For this reasons an increased IOP and the glaucomatous cup can be associated with the forms defined OC-RVO.

In the RVO group a statistically significant difference was found $(\mathrm{p}<0.05)$ in four parameters in the comparison between the OC group and the AV group: Cup Area, Cup/Disc Ratio, Rim Volume and Cup Shape Measure, in seven parameters comparing the OC group and the NONHS group: Cup Area, Cup/Disc ratio, Height Variation Contour, Rim Volume, Cup Shape Measure, Mean RNLF thickness and RNFL Cross Sectional Area (CSA).

In the RVO with POAG group we note a statistically significant difference similar to the previous one concerning the comparison between the OC group and the AV group. We note a significant difference in five parameters in the comparison between the OC group and the NONHS group:
Cup Area, Cup/Disc ratio, Rim Volume, mean Cup Depth and Cup Shape Measure.

In conclusion, the classification on the basis of the occlusion site could positively contribute in lowering the confusion that may arise from the classical subdivision between central vein and branch occlusions, helping in reducing the erroneous diagnosis, creating a logic support on which research on causal factors and the possible associations with the risk factors can be based on.

\section{CONFLICT OF INTEREST}

The authors confirm that this article content has no conflict of interest.

\section{ACKNOWLEDGEMENTS}

Declared none.

\section{REFERENCES}

[1] Hahn P, Fekrat S. Best practices for treatment of retinal vein occlusion. Curr Opin Ophthalmol 2012; 23(3): 175-81.

[2] Tuulonen A, Airaksinen PJ, Erola E, et al. The finnish evidencebased guideline for open angle glaucoma. Acta Ophthalmol Scand 2003; 81: 3-18.

[3] European Glaucoma Society. Terminology and Guidelines for Glaucoma. $3^{\text {rd }}$ ed. Dogma Editrice, Savona (Italia) 2008.

[4] Chauhan BC, Mikelberg FS, Balaszi AG, Le Blanc RP, Leske MR, Trope GE. Canadian glaucoma study: risk factors for the progression of open angle glaucoma. Arch Ophthalmol 2008; 126(8): 1030-6.

[5] Kim MJ, Woo SJ, Park KH, Kim TW. Retinal nerve fiber layer thickness is decreased in the fellow eyes of patients with unilateral retinal vein occlusion. Ophthalmology 2011; 118(4): 706-10.

[6] Sonsjö B, Dokmo Y, Krakau T. Disc haemorrhages, precursors of open angle glaucoma. Prog Retin Eye Res 2002; 21(1): 35-56.

[7] Sonnsjö B, Krakau CE. Arguments for a vascular glaucoma etiology. Acta Ophthalmol 1993; 71(4): 433-44.

[8] Beaumont PE, Kang HK. Clinical characteristics of retinal venous occlusions occurring at different sites. Br J Ophthalmol 2002; 86(5): 572-80

[9] Iester M, Mikelberg FS, Courtright P, Drance SM. Correlation between the visual field indices and Heidelberg retina tomograph parameters. J Glaucoma 1997; 6(2): 78-82.

[10] Strahlman ER, Quinlan PM, Enger C, Elman MJ. The cup-to-disc ratio and central retinal vein occlusion. Arch Ophthalmol 1989; 107(4): 524-5.

[11] Mansour AM, Walsh JB, Henkind P. Optic disc size in central retinal vein occlusion. Ophthalmology 1990; 97(2):165-6.

[12] Ravalico G, Battaglia Parodi M. Cup/disc ratio in branch retinal vein occlusion. Ophthalmologica 1991; 203(2): 53-6.

[13] Wanichwecharungruang B, Jaksataphorn P, Yuttitham K, Vanichvaranont S, Harncharoen K. Optic disc area and diameter of the central retinal vein occlusion fellow eyes, determined by optical coherence tomography. J Med Assoc Thai 2011; 94(Suppl 2): S7680 .

[14] Barnett EM, Fantin A, Wilson BS, Kass MA, Gordon MO. Ocular Hypertension Treatment Study Group. The incidence of retinal vein occlusion in the ocular hypertension treatment study. Ophthalmology 2010;117(3): 484-8.

[15] Citirik M, Sonmez K, Simsek T, Unal M. Optic disk analysis with heidelberg retina tomography in patients with branch retinal vein occlusion. Retina 2012; 32(5): 985-9.

[16] Beaumont PE, Kang HK. Cup-to-disc ration, intraocular pressure and primary open angle glaucoma in retinal venous occlusion. Ophthalmology 2002; 109(2): 282-6

[17] Călugăru D. Risk factors in central retinal vein occlusion. Otalmologia 2001; 55(2): 27-37.

[18] Glacet-Bernard A, Coscas G, Pournaras CJ. Preconceived ideas, paradoxes, and new concepts in retinal vein occlusion. $\mathrm{J}$ Fr Ophtalmol 2011; 34(8): 583-8. 
[19] Hayreh SS, Hayreh MS. Hemi-central retinal vein occulsion. Pathogenesis, clinical features, and natural history. Arch Ophthalmol 1980; 98(9):1600-9.

[20] Lindblom B. Open angle glaucoma and non-central retinal vein occlusion--the chicken or the egg? Acta Ophthalmol Scand 1998; 76(3): 329-33.
[21] Dobree JH. Venous obstruction and neovascularization at the disc in chronic glaucoma. Trans Opthal Soc UK 1957; 77: 229-37; discussion 237-8.

[22] Jung JJ, Baek SH, Kim US. Analysis of the causes of optic disc swelling. Korean J Ophthalmol 2011; 25(1): 33-6.

(C) Actis et al.; Licensee Bentham Open.

This is an open access article licensed under the terms of the Creative Commons Attribution Non-Commercial License (http://creativecommons.org/licenses/by$\mathrm{nc} / 3.0 /$ ) which permits unrestricted, non-commercial use, distribution and reproduction in any medium, provided the work is properly cited. 